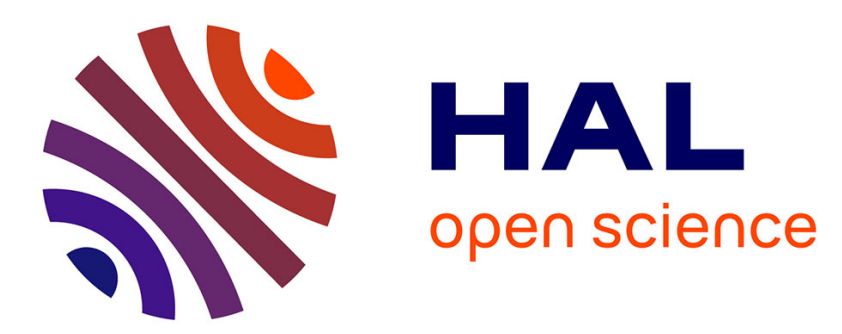

\title{
Optimization through Coupling Learning Capability and Imitation Strategy in a Multi-Robot System
} Abdelhak Chatty, Philippe Gaussier, Ilhem Kallel, Adel M.Alimi

\section{To cite this version:}

Abdelhak Chatty, Philippe Gaussier, Ilhem Kallel, Adel M.Alimi. Optimization through Coupling Learning Capability and Imitation Strategy in a Multi-Robot System. IEEE International Conference on Individual and Collective Behaviors in Robotics, Dec 2013, Tunisia. pp.1-6. hal-00955967

\section{HAL Id: hal-00955967 https://hal.science/hal-00955967}

Submitted on 6 Mar 2014

HAL is a multi-disciplinary open access archive for the deposit and dissemination of scientific research documents, whether they are published or not. The documents may come from teaching and research institutions in France or abroad, or from public or private research centers.
L'archive ouverte pluridisciplinaire HAL, est destinée au dépôt et à la diffusion de documents scientifiques de niveau recherche, publiés ou non, émanant des établissements d'enseignement et de recherche français ou étrangers, des laboratoires publics ou privés. 


\title{
Optimization through Coupling Learning Capability and Imitation Strategy in a Multi-Robot System
}

\author{
Abdelhak Chatty ${ }^{1,2}$,Philippe Gaussier ${ }^{2}$, Ilhem Kallel $^{1}$, and Adel M. Alimi ${ }^{1}$ \\ ${ }^{1}$ REGIM: REsearch Groups on Intelligent Machine \\ National School of Engineers (ENIS), Sfax University, Sfax, Tunisia \\ ${ }^{2}$ ETIS: Neuro-cybernetic team, Image and signal Processing \\ National School of Electronics and its Applications (ENSEA), Cergy-Pontoise University, Paris, France \\ \{abdelhak_chatty, ilhem.kallel, adel.alimi\}@ieee.org, Gaussier@ensea.fr
}

\begin{abstract}
In this paper, we present the interest of coupling learning capability and imitation strategy on individual and population levels in the field of Multi-Robot System. Particularly, we show that in an unknown environment adding a simple imitation capability to our bio-inspired architecture leads to a positive effect in the improvement the overall performance of the whole system. Indeed, our motivations is to optimize the robots goals discovery time and to improve the survival rate of agents. To analyze and validate our hypothesis, a series of experiments has been performed with and without a low level imitation strategy in a simulated multi-robot system. We will conclude with robotics' experiments which will feature how our approach applies accurately to real life environments.
\end{abstract}

\section{INTRODUCTION}

Several benefits can be expected from imitation capability [1], [2], [3] to improve the performance of the multirobot system and to share partial knowledge between robots. The imitation strategy can be considered as a powerful tool for autonomous robots to learn and discover new tasks and places. Coupling learning and imitation is an intuitive and natural method, it is not only the tool for learning but also a way to speed up the learning process. Therefore modern robotics considers imitation as a powerful behavior that enables learning by observation [4], [5]. A series of experiments has been performed to validate that the imitation strategy improves multi-robot system [6], [7], [8], [9], [10], [11].

In our case, in addition to robots, our simulated environment can contain three goals (G1, G2 and G3), and our real environment contain two goals (G1 and $\mathrm{G} 2)$. The role of goals is linked with robot's life-cycle. Actually, our robots are designed to be mortal. A robot's life-cycle is driven by a set of essential needs which are to be regularly satisfied by a corresponding goal. When a robot finds a particular type of goal, the associated need(s) are immediately fulfilled. Then follows a satisfaction decrease step during which the robot can freely move into the environment while the need for this goal type increases (see Fig.1). If the robot fails to come back to the location of a goal of this type before the corresponding satisfaction level reach a critic low threshold, it dies. The level of each type of need is internally represented by an essential variable, $e_{i}(t)$ whose value is in $[0 ; 1]$ and varies with time according to eq. 1 .

$$
d e_{i} / d t=-\alpha_{n} e_{i}(t)
$$

where $\alpha_{n}$ represents the decreasing rate of the essential variable. A value of 1 indicates a fulfilled need and a value of 0 means that the robot died by lack of the corresponding goal type.

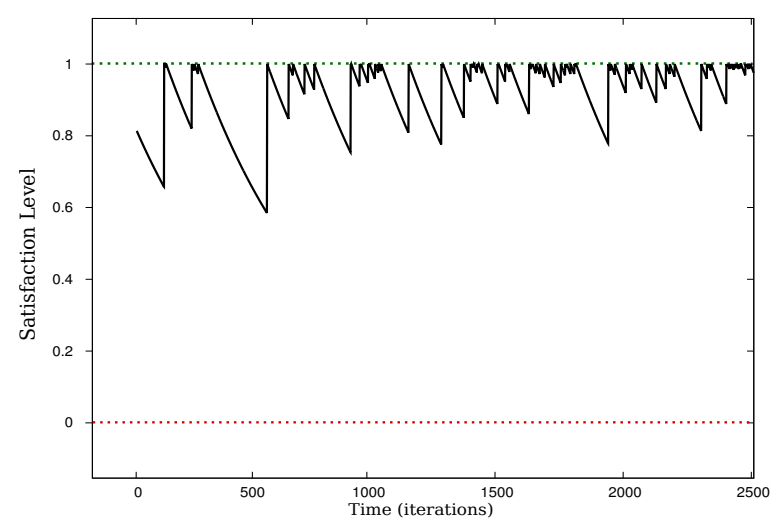

Fig. 1. Essential needs satisfaction levels undergo an exponential permanent decrease and are fullfilled when the robot achieves goals

Hence, the main individual goal of our robots is to sustain the satisfaction for all their essential needs at the highest possible level to ensure their own survival. Robots are thus endowed with navigation objectives, which first implies exploring the environment to find goals deposits, then frequently coming back to those deposits to refuel. Thus, in order to maintain the satisfaction level of our multi-robot system, keep them alive and optimize their planning time, it would be interesting if the robots were able to share the positions of the goals for the other robots.

The aim of this work is to show the positive feedback of coupling learning and imitation in a multi-robot system for navigating and planning task. Particularly, we analyze the association of the concept of a very low level imitation with our bio-inspired architecture which allows the learning and the building of the cognitive map.

The remainder of this paper is organized as follow: in section 2 the bio-inspired architecture is presented. Section 3 describes the behaviors of a robot. The imitation process is described in sections 4. Finally, section 5 and 6 are devoted to the description of the positive effect of the coupling of the imitation strategy and the learning capability in a multi-robot system. 


\section{THE ROBOT'S BIO-INSPIRED ARCHITECTURE}

It's true that there is a huge amount of work on graph theory. However, starting from neurobiological hypotheses on the role of hippocampus in the spatial navigation, we propose a biologically plausible model. Indeed, our architecture takes inspiration from the model presented in [12] which describes the role of the hippocampus. The Entorhinal Cortex (EC) which is the main input to the hippocampus receives signals from associative cortical areas than filters and merges this multimodal information in order to transfer it to the pyramidal cells (CA3) and to the dentate gyrus (DG). The DG puts the signals together in a temporal hierarchy which is later on retransmitted on CA3 cells. This temporal hierarchy allows CA3 to be aware of past events and put them in correspondence with present events, therefore the temporal hierarchy behaves like an associative memory through stocking possible transitions between these events. The recognition of the ongoing sequence happens at the level of CA1 using EC and CA3 information. It also extends to the prefrontal cortex (PFC) to serve the higher levels of cognitive processes.

In our case, the signals provided by the EC are solely spatial and consistent with spatial cells' activities [13]. In order to select only the cell with the strongest response at a specific location, spatial cells' activities are submitted to a Winner-Take-All competition. We subsequently speak about the current location by indicating the spatial cell which has the highest activity at a given location. The temporal function at the level of the DG is reduced to the mere memorization the past location. The acquired association at the level of the CA3 pyramidal cells is then the transition from a location to another aside from all information concerning the time spent to carry out this transition. Once the association from the past location and the new one is learned, every new entry will reactivate the corresponding memory in the DG. A schematic view of our architecture is shown in Fig. 2.

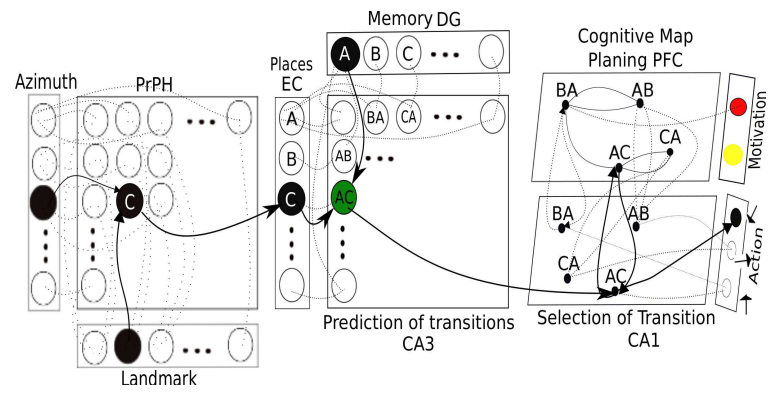

Fig. 2. Model for the building of a robot's cognitive map [14].

\section{A. Place celles recognation}

Our robots are shipped with a second independent camera which scan the visual area in front of them with a 180 degrees angular extent in search of known key points. Referred to as landmarks, a robot, once recognized a set of them, can associate each one with its perceived azimuth and use them as a position reference frame. The invariance to robot orientations is achieved by turning the azimuth absolute, using a compass for each robot. If enough landmarks have been learned, the perception of a particular set of landmarks codes for a unique location in the world. Thus, a robot is able to know, at any time, its position with respect to its own landmarks reference frame, allowing him to recognize already visited places so as to visually map its environment. Once detected, landmarks/azimuths are learned by means of a neural map which is able to store and recall landmarks/azimuths patterns in order to recognize previously visited places. When a robot discovers a new place, it first check if the perceived landmarks/azimuths pattern is close to one of the memorized places patterns. If one of the previously learned patterns is similar enough to the current perceived one according to a fixed vigilance threshold parameter, the associated neuron in the map is activated to reflect that the robot recognized this already visited place. If none of the learned patterns is similar enough, the current location is considered as an unknown place and the perceived landmarks azimuths pattern is learned by recruiting a new neuron which will respond to this place if the robot comes back here in the future. We called these neurons Place Cells (PC) [13]. Indeed, according to neurobiological hypothesis which highlights the importance of the hippocampus in the spatial navigation differents authors [13], [15], [16], [17] has brought to light special cells in the rodent's hippocampus that fire when the animal is at a precise location. These neurons creates a map which progressively cover the entire environment as the robot explores it (see Fig. 3).

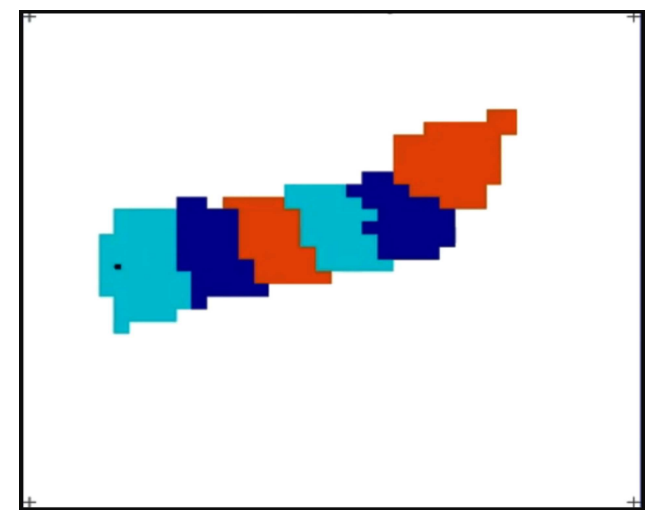

Fig. 3. Learning and responding to distinct landmarks/azimuths patterns, PCs act as a subjective positional reference frame (here, each colored patch represents the influence area of a unique PC)

Th election of the currently responding PCis the result of a neural competition process in which the PC of maximum activity is chosen to represent the current place. The PCs' activation function is chosen to be higher if the similarity between a PC's associated landmarks/azimuth pattern and the currently perceived one is higher. Hence, at any time, the winner PC, which is always associated with the most similar pattern, becomes active. Therefore a robot can recognize a known place and locate itself in its own neural map thanks to a unique firing PC. Generally, the vigilance parameter, which controls the granularity level of the neural map, is set to a value close to 1 (often around 0.98 ) to allow cells to respond only in a narrow neighborhood (referred to as influence area). Inspired from neural structures found in rodents, PCs map are the entry point for the bio-inspired neural navigation architecture, since it allows to link a robot's location in the world to behaviors and actions performing. 


\section{B. Building of the cognitive map}

To build a path from the current location to the another place, the cognitive map of course relies on the PCs map. But instead of directly drawing a course across adjacent PCs, as in gradient following strategies, the planning process uses a learned graph of Transition Cells (TCs) [18]. In other words, we do not directly use the PCs to navigate, plan or construct a map we rather use the TCs. A TC codes a spatiotemporal transition between two PCs successively winning the competition, respectively at time $\mathrm{t}$ and $\delta t$. The reason behind using transition cells is that their association with an action is univocal and quite straightforward. Thus, there is no need of an external algorithm to extract the action from the cognitive map. The TCs are learned and reinforced each time the robot move from the transition cell's origin place cell to its target place cell. The set of all the connected TCs and PCs forms a graph which constitute in itself a non-cartesian cognitive map (see Fig.4).

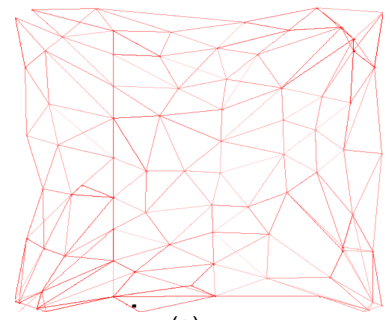

(a)

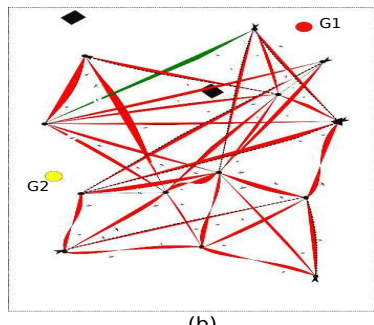

(b)
Fig. 4. The building of the cognitive map which is related to the own perception of the robot. (a) the cognitive map of a simulated robot at 20000 time steps, (b) The cogntive map of a real robot after 30 min of learning.

A passive forgetting factor (similar to what can be achieved in swarm-intelligence systems, except that shared knowledge does not use the physical space [19], [20] as repository but uses individual cognitive maps instead) is set on the transition cells through the synaptic weights between couples of connected transition cells (which can vary from 0 - fully forgotten to 1 - just visited). Transition cells which were recently visited are better remembered and motivation to take the corresponding edge is higher than transition cells which have not been for a long time. The cognitive map is then a learned neural graph of weighted transition cells which reflect the relative remembering of visited places, with access, through the underlying PCs map, to the associated motivations. During the exploration of the environment, the cognitive map is gradually created when the robot moves from one place to another. The equations governing learning in the cognitive map are given below eq. 2 and 3 :

$$
\begin{gathered}
\frac{d W_{i j}^{C C}(t)}{d t}=T(t) \cdot\left(\left(\gamma-W_{i j}^{C C}\right) \cdot X_{i}^{C}(t) \cdot X_{j}^{C}(t)-W_{i j}^{C C}(t) \cdot\left(\lambda 1 \cdot X_{j}^{C}(t)-\lambda 2\right)\right) \\
\frac{d W_{i j}^{M C}(t)}{d t}=S(t) \text { for }_{i, j}=\text { arg_zax }_{k, l}\left(X_{l}^{C}(t) \cdot X_{M}^{K}(t)\right)
\end{gathered}
$$

$T(t)$ is a binary signal ( 0 or 1$)$ activated when a transition occurs (moving from one place to another). This signal controls the learning of recurrent connections on the cortical map $W^{C C}$ . $\gamma$ is a parameter less than 1 which regulates the distribution of motivation activity on the map. $\lambda 1$ and $\lambda 2$ are respectively parameters of active and passive forgetting. $S(t)$ is a signal associated to the goal satisfaction. This signal controls the learning of synaptic connections between neurons in $W^{M C}$ motivations' activity $X^{M}$ and neurons of the cognitive map of activity $X^{C}$. After having explored the environment, the robots are in a position of predicting the locations directly reachable in each situation and to perform a gradient ascent according to their cognitive maps and their associated drives.

\section{ROBOT'S BEHAVIORS}

Our robots denotes a independent entity shipped with sensors and actuators, which is then able to perceive stimuli from the environment and act accordingly. A robot is able to perceive objects only in a local neighborhood in relation with the accuracy radius of its sensors. Thanks to this perceptionaction loop, which implies sensing from the environment, computing/learning, acting, sensing back, etc... a robot is to freely explore its environment and find a way to survive against predefined constraints in the environment and on the population.

\section{A. Associating motivations to places of interest}

Because of the link between essential needs satisfaction and presence of goals at particular places, all locations don't have an equal interest for the robots. Indeed, a good navigation strategy mostly implies refueling as often as possible, thus following the shortest courses which optimally link all goals types. To approach this global objective, robots can associate motivations to particular PCs. Hence, when a goal is discovered at an unknown place or is found at a previously free location, the robot tags this place as potentially interesting by setting a motivation for the goal's type to the corresponding PC. This labeling by motivations results in a homogeneous interest landscape which can guide the movements of the robot towards the refueling locations. Thus, when the need for a given goal type reaches a given threshold, the robot switches from an exploration behavioral state to a motivated state, PCs which are associated to the corresponding drive are stimulated and fire in their turn, in order to make target locations poppingout. Then a path is planed through the neural map to reach the interesting target places.

\section{B. Propagating motivations and selection of the shortest path}

Once learned, the cognitive map can be used to plan paths to the firing target cells by propagating their activities through the weighted graph of transition cells (see Fig. 5). Learning TCs is very useful when propagating the motivations to reach a target. As propagating motivation though the PCs map gives only one intermediate target $\mathrm{PC}$ from each intermediate origin PC, workarounds and complex sinuous paths in case of obstacles can't be found (topology is not explicit and thus the robot can't guess that another longer workaround can get it out from a blocking situation). In contrast, propagating motivation directly in an explicitly learned graph of transitions allows solving complex mazes by neglecting edges which lead to no valid solution.

Propagation is carried out using a simple convergent iterative algorithm which step by step floods the network with the activities of firing motivation sources. While spreading through the graph, neural activity is multiplied with the edges' 
weights (in a neural network classic feed-forward manner) and each transition cell takes the maximal activity of the incoming synaptic signals. After several iterations, the map converges to a stable state with a gradient like activity profile from high activity motivation sources neighboring cells to low activity forgotten or distant cells. By following this increasing activation profile, moving from the current $\mathrm{PC}$ to the target $\mathrm{PC}$ of the maximum activity out-coming TC, the robot can reach the PCs which fired the motivational drive. Using such a propagation method allows solving motivation drives concurrency, insofar as multiple propagation sources will progressively merge until reaching the current $\mathrm{PC}$. What is interesting is that since each time a motivation passes through a TC, it is weaken by the synaptic weights. As a consequence, motivation which comes from closer places will be favored, and forgetting will be taken into account. So, the robots will choose the shortest path to reach the goal provided that the path to reach it has been taken recently.

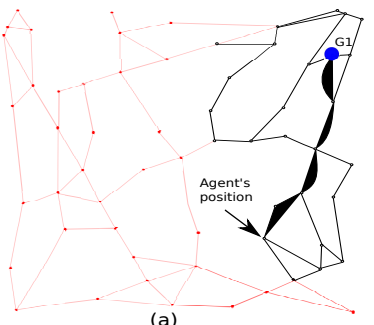

(a)

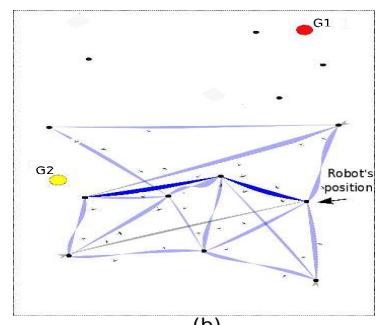

(b)
Fig. 5. Once propagated, motivations from needed goals locations spread into an activity gradient on transition cells. Paths can be plan by following this landscape. (a) the selection of the shortest path by a simulated robot (b) the selection of the shortest path by a real robot.

\section{Planning vs. Exploration}

When the satisfaction level for all essential needs is high (above the threshold), the robot considers itself as globally satisfied, and no motivational drive is triggered. But if even only one essential level falls under the threshold, a motivation for the corresponding goal type is fired and propagated to refuel immediately. If more than one need crosses the threshold at the same time, there can be more than one drive simultaneously triggered. When a motivation is triggered, the robot enter the planning mode, while, when there are no motivation, the robot stays in a quiet random exploration mode. Metaphorically, the frequency of motivations trigerring to exploration ratio is referred to as stress. Afterwards, constructing a satisfactory path is then the role of the motivation propagation process in the cognitive map's.

\section{THE IMITATION PROCESS}

We describe in this section a very simple architecture for imitation in a navigation perspective. At the social level, robots can take advantage of the ability to imitate one another using simple robot-following strategies to transmit parts of one robot's cognitive map to another's, leading to some kind of naturally distributed knowledge. The proposed model is based on dynamical interactions among mobile robots. Our aim is to provide limited capabilities to a mobile robot to interact dynamically with other robots by following their current direction of movement.
In simulated robots, we implemented and studied a simple imitation strategy based on the azimuth: if a single robot is visible, it becomes the chosen imitation target; if several robots are visible, then the chosen target is the closest one to the direction of the robot which tries to imitate. The decision of imitation is controlled by a probabilistic function (see eq. 6): the probability of imitation decreases when the number of discovering of different kind of goals $N_{D}$ increases, where $\eta$ and $\rho$ are positive values.

$$
\operatorname{Pr}_{(\text {Imitation })}=\eta \exp ^{-\rho N_{D}}
$$

In real robots, perceived motions in the visual field of a robot are estimated by a classical optical flow algorithm [21]. If the perceived motion is in upward direction, it is considered as positive activity, on the other hand, downward motion is accumulated as negative activity. Similarly, motion on the left direction leads to positive activity while movements on the right direction are considered as positive activity.

\section{IMITATION BY A SIMULATED MULTI-ROBOT SYSTEM}

The behaviors of our simulated robots are not deterministic because they do not always provide the same results on several tests with the same parameters. In order to obtain complete results, we conducted ten tests with 60 simulated robots for each experiment until 20000 time steps (the number of tests is determined by the statistical Fisher test).

To better understand the imitation behavior of simulated robots, fig. 8a shows that the robots represented by triangles, started to move randomly in the environment.

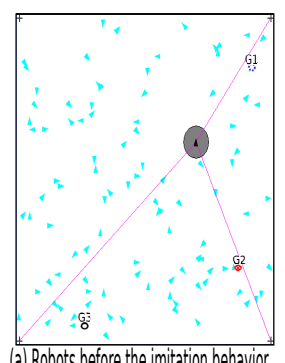

(a) Robots before the imitation behavior

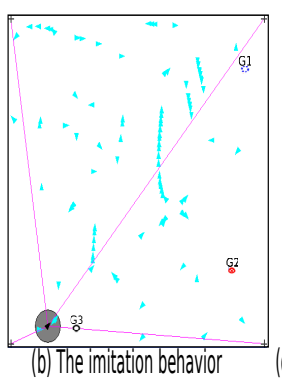

(b) The imitation behavior

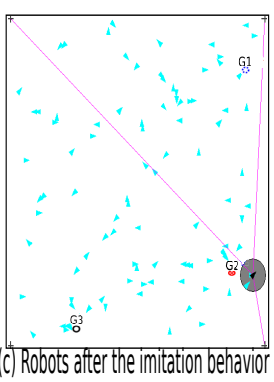

Robots after the imitation behavior

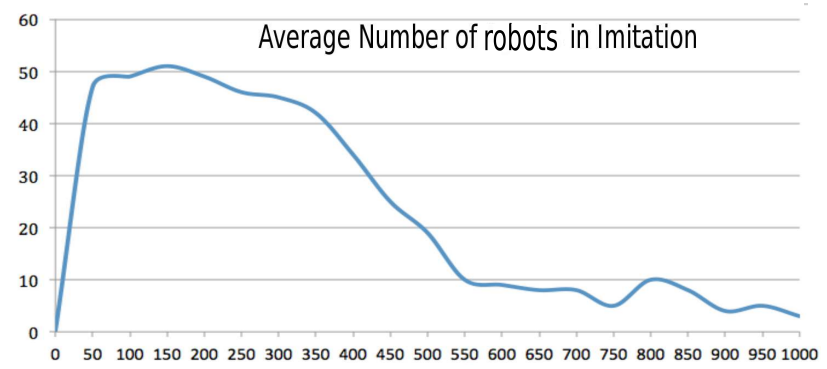

Time steps

(d)

Fig. 6. The evolution of the robots' number in imitation through 1000 time steps.

Due to the imitation behavior fig. $8 \mathrm{~b}$ shows that subgroups of robots appeared in the environment. According to the curve (d), this phase coincides with an increase in the number of robots in imitation. Indeed, the robots try to follow each other so as to localize and learn the goals' positions. We can also 
see that some subgroups, have succeeded to discover the goal G1 and G2 and to learn their positions. After discovering all kinds of goals the probability of the imitation will decrease (see fig. 8c). Thus, the number of robots in imitation shown by the curve (d) also decreases, since robots are now able to return to the goals on their own cognitive map.

\section{A. Improvement of the survival Rate}

To study the influence of the imitation strategy on the survival rate of the populations, we used the same environment and launched 20,30, 40 then 60 robots, and counted the number of robots that survived, or died for not having found all of the three types of goal. The results show that adding an imitation capability can dramatically enhance the survival rate of the population from $45,66 \%$ (without imitation behavior) to $60,64 \%$ (with imitation). Indeed, coupling imitation behavior with the cognitive map allows robots to discover and to learn the position of the goals in the environment more rapidly

\section{B. Optimization of robots' goals discovery time}

Thanks to the imitation strategy, our simulated robots are able to find the goals and to optimize their discovering time from 1683 time steps (5 minutes) without imitation to 505 time steps (1.5 minutes) with imitation strategy (see Fig. 9). Through the imitation behavior we show that the robots can more rapidly learn the position of the goals and satisfy their needs. Thus, the robots would be able to improve the performance of the whole system.

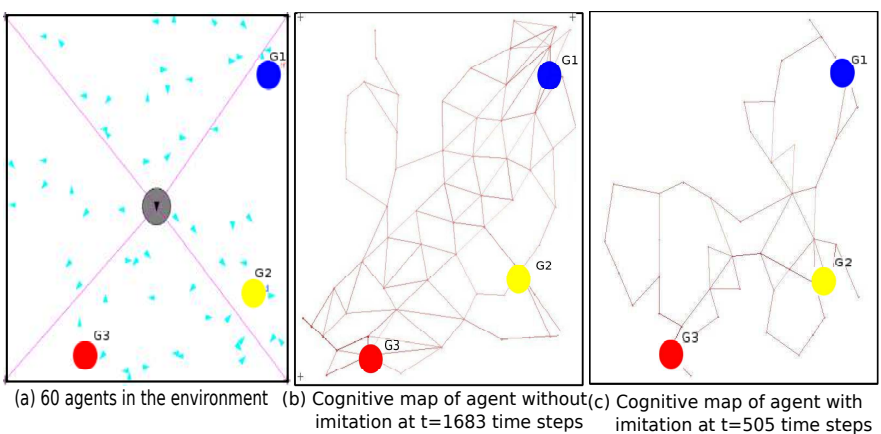

Fig. 7. The positive feedback of the imitation strategy in a simulated multirobot system. Fig. (a) presents 60 robots and 3 golas (G1,G2 and G3) in the environment. Fig. (b) shows that without imitation simulated robot_1 is able to find the three goals after 1683 time steps (5 minutes). However, in Fig. (c), the imitation strategy allows simulated robot 2 to reach the goals after 505 time steps ( 1.5 minutes) which influences the shape of the cognitive maps. Indeed, with imitation, the shape of the cognitive map is more simple than without imitation which allows the robot to better optimize its planning time.

\section{IMITATION BY A REAL MULTI-ROBOT-SYSTEM}

To study the influence of imitation on the behavior of the robots, we used three robots. Two leader robots (LR) were already learned the position of the two goals and created their cognitive map, along with one reactive imitator robot (IR) which uses just an imitation strategy. Fig. 10 (a) shows the IR tries to follow the LR. Indeed, in Fig. 10 (b), (c) and (d) the IR performs an imitation behavior which allows it to find the first goal G1 by following the perceived LR.

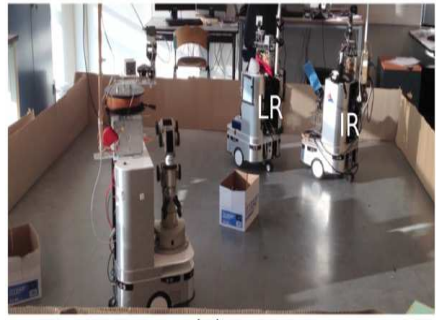

(a)

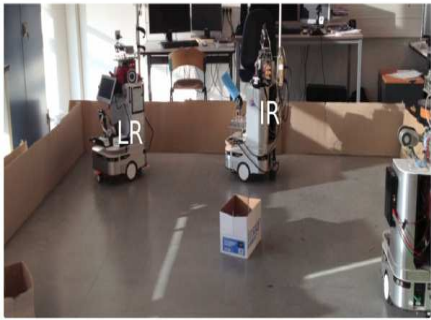

(c)

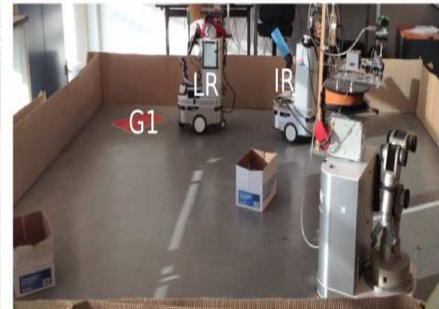

(b)

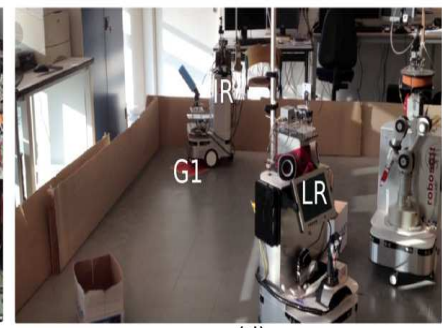

(d)
Fig. 8. The discovery of the 1 goal. The experiment is done with three robots in the same environment, imitator reactive robot (IR) and two leader robots (LR), G1 and G2 are the two goals. By imitating the trajectory of the LR, the IR was able to find the first goal.

To discover the second goal, Fig. 11 (a) shows the IR tries to follow the LR. In Fig. 11 (b) and (c) the LR changed its direction and it became an obstacle for the IR. Thanks to the avoidance of the dynamical obstacols, the LR was able to find the second goal. Thus, coupling the imitation behavior with the avoidance of obstacols allows the IR to find its goal.

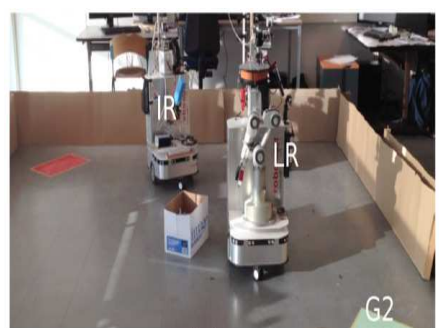

(a)

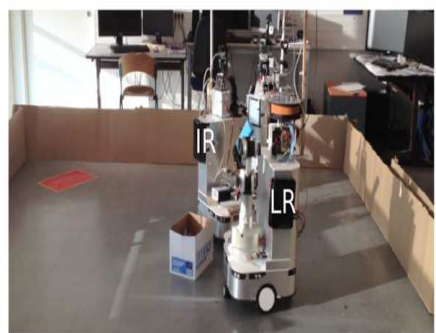

(c)

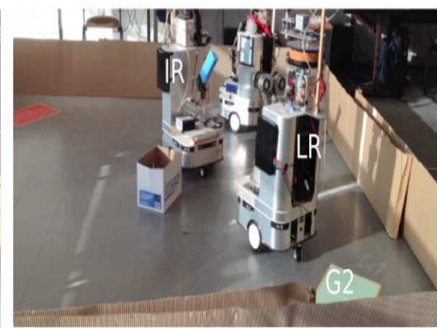

(b)

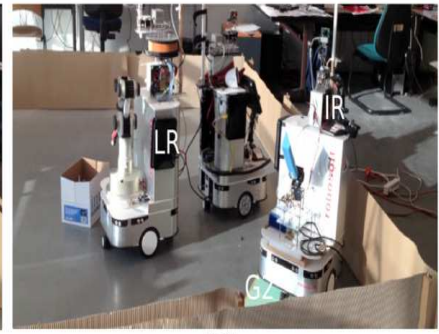

(d)
Fig. 9. The discovery of the 2 goal. Thanks to the coupling of the imitation behavior with the avoidance of obstacols, IR was able to find its goal.

\section{A. The effect of imitation on the goals discovery time}

In order to compute the discovering time of both goals in the environment, two different experiments have been tested according to the following scenarios: a single reactive robot in the environment without imitation capability and the same 
reactive robot with imitation capability along with a cognitive robot that already know the locations of the goals in the environment. To find the average time for the goal's discovery in each scenario, we conducted several tests. The number of tests to take the average value is determined by the Fisher test.

The average time to find the two goals for each scenario is plotted in Fig. 12. Thanks to the imitation strategy the imitative reactive robot takes less time to find both goals (G1 and G2) by following the cognitive robot, its accumulative average time is about 5 minutes ( 2 min. for G1 and 3 min. for G2). However, the reactive robot of the first scenario without imitation strategy take much more time (about 3 times more) to discover goals. Indeed, the accumulative average time is 22 minutes $(6 \mathrm{~min}$. for $\mathrm{G} 1$ and $16 \mathrm{~min}$. for $\mathrm{G} 2$.

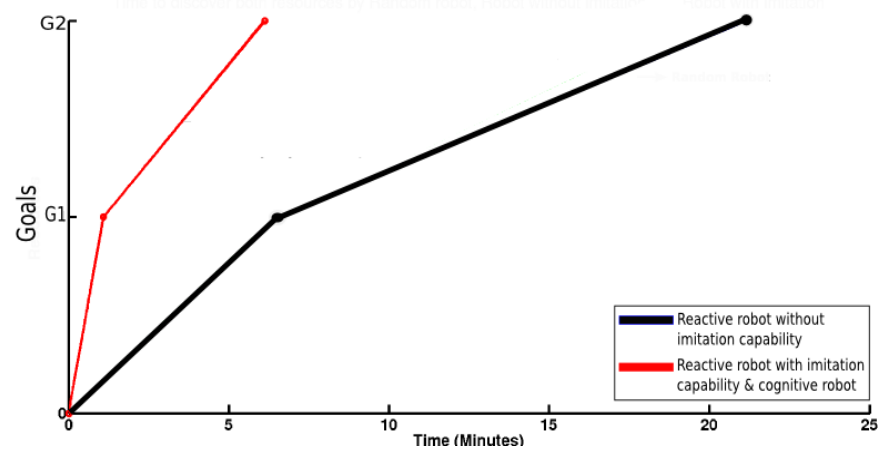

Fig. 10. The average time of the goals discovery. The red curve shows the time to discover the goals by a reactive robot with imitation capability along with a cognitive robot which already knows the goals. The black curve describes the time taken by a single reactive robot without imitation capability to explore the goals.

\section{CONCLUSION}

In this paper, we highlighted the importance of coupling the imitation strategy with learning capability which leads to the improvement of the performance of the whole multi-robot system. This coupling boosts the capability of robots to adapt to an unknown environment, allows to solve the navigation task among various targets and finally optimizes the time to explore the goals. In the experiments with multi-robot system, we noted several situations where performance of the system decreases when the $I R$ tries to follow the $L R$ without knowing that the $L R$ goes to the goals or not. Indeed, the $I R$ may follow the $L R$ to find uninteresting places. As prospects, the integration of emotional mechanism seems necessary to improve the imitation strategy [22]. We are slso trying to study the effect of the imitation and the learning in a cognitive multirobot system in the field of agregation of objects.

\section{ACKNOWLEDGMENT}

The authors would like to thank the financial support of the Tunisian General Direction of Scientific Research and Technological Renovation (DGRSRT), under the ARUB program 01/UR/11 02, the Institut Français de Tunisie, the INTERACT french project referenced ANR_09_CORD_014, the NEUROBOT french project referenced ANR-BLAN-SIMI2L2-100617-13-01 and DIGITEO project AUTO EVAL.

\section{REFERENCES}

[1] P. Gaussier, S. Moga, J. P. Banquet, M. Quoy, and N. E. Modelisations, "From perception-action loops to imitation processes: A bottom-up approach of learning by imitation," 1997.

[2] S. Schaal, J. Peters, J. Nakanishi, and A. Ijspeert, "control, planning, learning, and imitation with dynamic movement primitives," in workshop on bilateral paradigms on humans and humanoids, ieee international conference on intelligent robots and systems (iros 2003), 2003, p. p1804.

[3] T. Chaminade, E. Oztop, G. Cheng, and M. Kawato, "From selfobservation to imitation: visuomotor association on a robotic hand." Brain research bulletin, vol. 75, no. 6, pp. 775-784, Apr. 2008.

[4] Y. Kuniyoshi, N. Kita, S. Rougeaux, S. Sakane, M. Ishii, and M. Kakikura, "Cooperation by observation - the framework and basic task patterns," in ICRA, 1994, pp. 767-774.

[5] S. Schaal, "is imitation learning the route to humanoid robots?" in trends in cognitive sciences, 1999, pp. 233-242.

[6] G. Hayes and J. Demiris, A robot controller using learning by imitation. Citeseer, 1994, pp. 198-204.

[7] M. Ollis, W. H. Huang, and M. Happold, "A bayesian approach to imitation learning for robot navigation," p. 709?714, 2007.

[8] J. P. Bandera, "Vision-based gesture recognition in a robot learning by imitation framework," Ph.D. dissertation, 2010.

[9] Y. Demiris and A. Billard, "Special issue on robot learning by observation, demonstration and imitation," pp. 254-255, 2007.

[10] A. Chatty, S. K. Hasnain, P. Gaussier, I. Kallel, P. Laroque, and A. M. Alimi, "Effect of low level imitation strategy on an autonomous multirobot system using on-line learning for cognitive map building," in ROBIO, 2012, pp. 1452-1457.

[11] A. Chatty, P. Gaussier, I. Kallel, P. Laroque, and A. M. Alimi, "Learning by imitation for the improvement of the individual and the social behaviors of self-organized autonomous agents," in ICSI (2), 2013, pp. 44-52.

[12] J. P. Banquet, P. Gaussier, J. C. Dreher, C. Joulain, A. Revel, and W. Gunther, "Spacetime, order and hierarchy in fronto-hippocamal system : A neural basis of personality. in cognitive science perspectives on personality and emotion." Elsevier Science BV, p. 123?189, 1997.

[13] J. O'Keefe and L. Nadel, The hippocampus as a cognitive map. Clarendon Press ; Oxford University Press, Dec. 1978.

[14] A. Chatty, P. Gaussier, I. Kallel, P. Laroque, and A. M. Alimi, "Adaptation capability of cognitive map improves behaviors of social robots," in ICDL-EPIROB, 2012, pp. 1-6.

[15] N. Burgess, J. G. Donnett, and J. O'Keefe, "Robotic and neuronal simulation of hippocampal navigation," University of Manchester, vol. 352, pp. 1361-6161, 1997.

[16] M. Milford and G. Wyeth, "Mapping a suburb with a single camera using a biologically inspired slam system," IEEE Transactions on Robotics, vol. 24, no. 5, pp. 1038-1053, October 2008.

[17] I. A. Bachelder and A. M. Waxman, "Mobile robot visual mapping and localization: A view-based neurocomputational architecture that emulates hippocampal place learning," Neural Networks, vol. 7, pp. 1083 - 1099, 1994.

[18] P. Gaussier, A. Revel, J. P. Banquet, and V. Babeau, "From view cells and place cells to cognitive map learning: processing stages of the hippocampal system." Biological Cybernetics, vol. 86, no. 1, pp. 15-28, 2002.

[19] M. Dorigo, E. Bonabeau, and G. Theraulaz, "Ant algorithms and stigmergy," Future Gener. Comput. Syst., vol. 16, no. 9, pp. 851-871, June 2000

[20] A. Chatty, I. Kallel, A. M. Alimi, and P. Gaussier, "Fuzzy counter-ant for avoiding the stagnation of multirobot exploration," in SMC, 2010, pp. 3358-3365.

[21] B. K. P. Horn and B. G. Schunck, "Determining optical flow," Artif. Intell., vol. 17, no. 1-3, pp. 185-203, 1981.

[22] C. Lola and G. Philippe, "Emotion understanding: Robots as tools and models," Oxford University Press, pp. 235-258, 2005. 